\title{
Perfil de automedicación en funcionarios de una industria farmacéutica
}

\author{
Raquel Cuevas, Lourdes Samaniego, Patricia Acosta, María Gloria Domenech, Gladys Lugo, *Gladys \\ Mabel Maidana
}

Universidad Nacional de Asunción. Facultad de Ciencias Químicas. Departamento de Farmacia. Paraguay

\author{
Cómo referenciar este artículo/ \\ How to reference this article:
}

Cuevas R, Samaniego $L$, Acosta P, Domenech MG, Lugo G, Maidana GM. Perfil de automedicación en funcionarios de una industria farmacéutica. Mem. Inst. Investig. Cienc. Salud. 2019; 17(1): 99-112

\begin{abstract}
RES U M E N
La automedicación es una práctica riesgosa cuyas consecuencias incluyen enmascaramiento de una enfermedad, aparición de reacciones adversas, interacciones medicamentosas, aumento de la resistencia a ciertos tipos de fármacos y farmacodependencia. El objetivo fue determinar el perfil de automedicación en funcionarios de una industria farmacéutica. Estudio descriptivo, observacional, con muestreo no probabilístico y por conveniencia realizado de octubre a noviembre del 2015, a 61 funcionarios que trabajan en una planta industrial farmacéutica. Las edades estaban comprendidas entre 20 y 30 años, en su mayoría mujeres. Consumían medicamentos $97 \%$ de los funcionarios. El $53 \%$ se automedicaba para patologías menores y $90,3 \%$ lo hacía según necesidad. Utilizaban mezcla de analgésicos y antiinflamatorios. Los motivos más frecuentes, $58 \%$ dijo no tener tiempo para ir al médico y $42 \%$ fue un familiar o amigo el que le sugirió el medicamento que debía consumir. De los que se automedicaban, el $74 \%$ consideraba que la publicidad no es importante para elegir un medicamento, el $81 \%$ utilizaba la información del prospecto. El $48 \%$ no recibía información sobre el medicamento en la farmacia y el $93 \%$ consideraba que la automedicación es peligrosa. La automedicación es una práctica común en la población de funcionarios estudiada y en su entorno social inmediato, a pesar de que la mayoría de la población entiende que la automedicación constituye una práctica riesgosa.
\end{abstract}

Palabras clave: automedicación, medicamentos, uso racional de medicamentos, farmacéutico.

\section{Self-medication profile of employees in a pharmaceutical industry}

\begin{abstract}
A B S T R A C T
Self-medication is a risky practice with consequences that include masking a disease, occurrence of adverse reactions, drug interactions, increased resistance to certain types of drugs and drug dependence. The objective was to determine the self-medication profile of employees in a pharmaceutical industry. A descriptive, observational study, with nonprobabilistic convenience sampling was conducted from October to November 2015, in 61 employees working in a pharmaceutical industrial plant. They were mostly women with ages between 20 and 30 years. Ninety seven percent of the used drugs, $53 \%$ selfmedicated for minor pathologies and $90.3 \%$ did it according to need using a mixture of analgesics and anti-inflammatories. Among the most frequent reasons, $58 \%$ said they did not have time to go to the doctor and $42 \%$ said that a family member or friend suggested the medication to be taken. Of the self-medicating employees, $74 \%$ believed that advertising is not important for choosing a drug, $81 \%$ used the information in the prospectus, $48 \%$ did not receive information about the drug in the drugstore and $93 \%$ considered that self-medication is dangerous. Self-medication is a common practice in the
\end{abstract}


population studied and in their immediate social environment, despite the fact that most of the population understands that self-medication is a risky practice.

Keywords: self medication, pharmaceutical preparations, rational use of drugs, pharmacist

\section{INTRODUCCION}

Los medicamentos constituyen la tecnología sanitaria más utilizada en la prevención y el tratamiento de enfermedades en todos los niveles de atención sanitaria, de ellos depende, en buena medida, la capacidad que hoy tiene la medicina de interrumpir o modificar el curso natural de las enfermedades, de prevenirlas, o, en cualquier caso, de hacer su peso más liviano.

La Organización Mundial de la Salud (OMS), define la automedicación como el uso de medicamentos incluidos productos herbarios y convencionales, por parte del paciente para tratar desórdenes o síntomas que el paciente reconoce, o el uso intermitente o continuado de una medicación prescrita por el médico para enfermedades o síntomas recurrentes o crónicos $^{(1)}$.

Este comportamiento está ampliamente extendido a nivel mundial, con cifras alrededor del $60 \%$ en países muy dispares económica y culturalmente ${ }^{(2)}$.

Los errores habituales de la automedicación se observan en los siguientes fármacos: analgésicos, antibióticos y antihistamínicos. Más del $50 \%$ de los medicamentos se prescriben, dispensan y venden de forma inapropiada y la mitad de los pacientes no los consumen de forma correcta. Su uso excesivo, insuficiente o indebido tienen efectos nocivos para los pacientes y constituye un desperdicio de recursos y más del $50 \%$ de los países no aplican políticas básicas para fomentar el uso racional de medicamentos ${ }^{(3)}$.

Prácticamente todo el mundo occidental tiene acceso a un medicamento que le calme el dolor de cabeza, que disminuye el estrés, su tendencia depresiva o la angustia vital que no falta en nuestros días por las tensiones de la vida actual ${ }^{(4)}$.

La automedicación constituye una práctica riesgosa cuyas consecuencias pueden ser, entre otras: enmascaramiento de una enfermedad (retrasando la asistencia médica en casos en los que es realmente necesaria), aparición de reacciones adversas e interacciones medicamentosas, aumento de la resistencia a agentes antimicrobianos, elección incorrecta de medicamentos y riesgo de abuso o farmacodependencia ${ }^{(5)}$.

Esta conducta puede ser en parte la respuesta más común a la enfermedad, sin embargo, se ha constituido en un problema creciente que tiene como contrapartida la posibilidad de abuso, con todos los riesgos que ello implica.

Conviene entonces diferenciarla de la autoprescripción, que se refiere al uso ilegal de medicamentos de venta bajo receta sin la prescripción médica correspondiente ${ }^{(1)}$.

La automedicación responsable requiere el uso de medicamentos seguros y eficaces, destinados a tratar condiciones que el paciente puede reconocer o estados patológicos crónicos o recurrentes luego de un diagnóstico médico inicial.

Los productos utilizados deben, además, incluir toda la información necesaria referente a cómo administrarlos, efectos adversos, interacciones medicamentosas, duración del tratamiento, etc. que favorezcan el uso adecuado por parte del paciente.

En este escenario la OMS ha destacado al profesional farmacéutico, lógicamente, como una figura clave en el equipo de salud para educar a la población en prácticas adecuadas y seguras de automedicación responsable ${ }^{(1)}$.

El farmacéutico mediante las intervenciones farmacéuticas ha ido demostrando a lo largo de los últimos años un papel en la mejora de resultados en la salud de los pacientes ${ }^{(6)}$.

En estudios sobre automedicación realizados en varios países como en España, en estudiantes universitarios de la ciudad de Murcia, la automedicación fue de $72,5 \%{ }^{(5)}$ y una cifra similar $(77,4 \%)$ en inmigrantes latinoamericanos adultos en Sevilla - España ${ }^{(7)}$. En otro estudio realizado en estudiantes de Venezuela ${ }^{(8)}$ la cifra fue de $87 \%$ y en Perú $^{(9)} 88 \%$.

Ante la falta de datos a nivel local relacionado a automedicación en funcionarios de industria farmacéutica, se planteó este estudio a fin de determinar el perfil de automedicación en esta población, para ello se determinaron los medicamentos más frecuentemente utilizados sin prescripción médica, los motivos y frecuencia de automedicación, describiendo la percepción del rol del farmacéutico en la dispensación de los medicamentos. 


\section{MATERIALES Y MÉTODOS}

Estudio observacional, descriptivo, de corte transverso con muestreo no probabilístico y por conveniencia realizado durante los meses de octubre a noviembre del 2015, a funcionarios que trabajan en una planta industrial farmacéutica.

Constituían la población 75 funcionarios con edades entre 20 y 60 años, de ambos sexos, de los cuales 61 aceptaron participar del estudio, previa información acerca de la investigación, y firmaron el documento de consentimiento informado.

Se utilizó un cuestionario estructurado anónimo con preguntas cerradas, de tipo elección múltiple, que fue administrado por los investigadores.

Las preguntas estaban orientadas a evaluar: a) si la automedicación es una práctica extendida en la población encuestada y en su entorno social inmediato y los motivos por los cuales se practicaba, b) los factores que influyen en la elección de un medicamento determinado y las categorías terapéuticas con las que mayormente se automedicaba la población bajo estudio, c) la percepción del encuestado del rol del farmacéutico como comunicador y formador en el uso racional de medicamentos y d) si la automedicación que practicaba el funcionario correspondía al concepto de automedicación responsable (¿hay diagnóstico médico previo? ¿lee el prospecto? ¿cumple con la duración del tratamiento indicada en prospecto? ¿se consumen, durante el tratamiento, alcohol o bebidas energizantes?).

El Comité de Ética de la Facultad de Ciencias Químicas de la Universidad Nacional de Asunción, evaluó y consideró que no se detectaron incompatibilidades éticas CEI-205/15.

\section{RESULTADOS}

Participaron del estudio 61 funcionarios, con predominio del sexo femenino (61\%) y de edades entre 20 y 30 años (67\%). Un $70 \%$ de los funcionarios llevaba más de 10 años trabajando en la industria farmacéutica.

Casi todos los funcionarios (97\%) manifestaron haber consumido medicamentos en los tres meses anteriores al estudio, de los cuales el $53 \%(n=31)$ se automedicaba para diferentes patologías menores.

Los 31 funcionarios que se automedicaban, principalmente eran del sexo femenino (58\%), de edades entre 20 a 30 años $(71 \%)$, nivel de instrucción universitaria $(61,3 \%)$ y con antigüedad en la empresa entre 1 a 10 años (65\%) (Tabla 1).

Tabla 1. Datos sociodemográficos de los que se automedicaron

\begin{tabular}{lc}
\hline \multicolumn{1}{c}{ Variables } & $\mathbf{n = 3 1}$ \\
\hline Sexo & $\mathbf{n}(\%)$ \\
Femenino & $18(58)$ \\
Masculino & $13(42)$ \\
Edad (años) & $\mathbf{n}(\%)$ \\
20 a 30 & $22(71)$ \\
31 a 40 & $6(19,3)$ \\
41 a 50 & $3(9,7)$ \\
51 a 60 & $0(0)$ \\
Nivel de instrucción & $\mathbf{n}(\%)$ \\
Primaria & $1(3,2)$ \\
Secundaria & $11(35,4)$ \\
Universitaria & $19(61,3)$ \\
Antigüedad en la empresa & $\mathbf{n}(\%)$ \\
Menor de un mes & $1(3)$ \\
2 meses -1 año & $9(29)$ \\
Más de 1 año -10 años & $20(65)$ \\
Más de 10 años & $1(3)$ \\
\hline
\end{tabular}


Entre los síntomas que los motivaban para la automedicación, el $68 \%$ refirió por dolor de cabeza y el $90,3 \%$ respondió lo hacía según necesidad, y que generalmente utilizaba una mezcla de analgésicos y antiinflamatorios (90,3\%). En cuanto a los motivos más frecuentes, $58 \%$ respondió que no tenía tiempo para ir al médico y que en la mayoría de los casos (42\%) fue un familiar o amigo el que le sugirió el medicamento que debía consumir. El $80,6 \%$ de los casos respondió que no se sintió mejor luego de automedicarse. El mismo porcentaje de funcionarios, refirió que cuando la enfermedad no mejoraba acudían al médico y que dejaba de utilizar los medicamentos cuando desaparecían los síntomas (Tabla 2).

Tabla 2. Respuesta respecto a la automedicación

\begin{tabular}{ll}
\hline Variables & $\mathbf{n = 3 1}$ \\
\hline Síntomas que motivaron\# & $\mathbf{n}(\%)$ \\
Dolor de cabeza & $21(67,7)$ \\
Dolor de garganta & $4(12,9)$ \\
Dolor de estómago & $3(9,7)$ \\
Otros & $8(25,8)$ \\
¿Con que frecuencia se automedica? & $\mathbf{n}(\%)$ \\
Diariamente & $2(6,5)$ \\
Mensualmente & $1(3,2)$ \\
Según necesidad & $28(90,3)$ \\
Me automedico más frecuentemente con: \# & $\mathbf{n}(\%)$ \\
Analgésicos /Antiinflamatorios & $28(90,3)$ \\
Antigripales & $5(16,1)$ \\
Antialérgicos & $1(3,2)$ \\
Vitaminas & $1(3,2)$ \\
Antiemético & $1(3,2)$ \\
Motivo de su automedicación & $\mathbf{n}(\%)$ \\
No tengo tiempo para ir al medico & $18(58)$ \\
Creo que no es necesario ir al medico & $13(42)$ \\
¿Quién le recomienda automedicarse? & $\mathbf{n}(\%)$ \\
Familiares y/o amigos & $13(42)$ \\
Farmacéutico & $9(29)$ \\
Otros & $9(29)$ \\
¿Se sintió mejor luego de automedicarse? & $\mathbf{n}(\%)$ \\
Si & $6(19,4)$ \\
No & $25(80,6)$ \\
¿Qué acción tomara? & $\mathbf{n}(\%)$ \\
Concurre al medico & $25(80,6)$ \\
Aumenta la dosis & $3(9,7)$ \\
Cambia de medicamento & $3(9,7)$ \\
¿Deja de automedicarse? & $\mathbf{n}(\%)$ \\
Cuando lo indica el prospecto & $5(16,1)$ \\
Cuando desaparecen los síntomas termina el medicamento & $25(80,6)$ \\
\hline
\end{tabular}

\# Marcaron más de una respuesta. 
Respecto al lugar de adquisición y fuente de información de medicamentos, el 74\% consideraba que la publicidad no es importante para elegir un medicamento, el $81 \%$ utilizaba la información del prospecto, y el 97\% compraba sus medicamentos de la Farmacia (Tabla 3).

Tabla 3. Lugar de adquisición y fuentes de información de medicamentos

\begin{tabular}{|c|c|c|}
\hline $\begin{array}{llll}\text { La publicidad es importante } & \text { para } \\
\text { medicamento: } & & & \\
\end{array}$ & elegir un & $\begin{array}{l}n=31 \\
n(\%)\end{array}$ \\
\hline 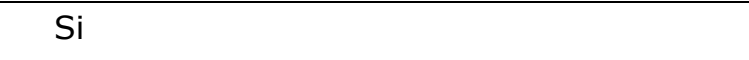 & & $8(26)$ \\
\hline No & & $23(74)$ \\
\hline ¿Utiliza la información del prospecto? & & $\begin{array}{c}n=31 \\
n(\%)\end{array}$ \\
\hline Si & & $25(81)$ \\
\hline No & & $4(13)$ \\
\hline En algunos casos & & $2(6)$ \\
\hline ¿De dónde compra los medicamentos? & & $\begin{array}{l}n=31 \\
n(\%)\end{array}$ \\
\hline Farmacia & & $30(97)$ \\
\hline Vía Publica & & $1(3)$ \\
\hline
\end{tabular}

Al ser consultados todos los participantes del estudio $(n=61)$ sobre la información que recibieron en la farmacia, el $48 \%$ dijo no recibir ningún tipo de información sobre el medicamento en la farmacia y el $98 \%$ consideraba importante que el farmacéutico le brinde información; el 93\% consideraba que la automedicación es peligrosa. Además el $89 \%$ de los participantes contaban con seguro médico y $9,8 \%$ respondieron que mezclaron bebidas alcohólicas con los medicamentos (Tabla 4).

Tabla 4. Percepción en cuanto al Farmacéutico y la automedicación de los participantes del estudio

\begin{tabular}{lr}
\hline $\begin{array}{l}\text { En la Farmacia: ¿recibe información sobre el } \\
\text { medicamento? }\end{array}$ & $\begin{array}{r}\mathbf{n = 6 1} \\
\mathbf{n}(\%)\end{array}$ \\
\hline $\mathrm{Si}$ & $20(33)$ \\
$\mathrm{No}$ & $29(48)$ \\
$\mathrm{A}$ veces & $12(19)$ \\
¿Considera importante que el Farmacéutico le & $\mathbf{n}(\%)$ \\
brinde información? & $60(98)$ \\
$\mathrm{Si}$ & $1(2)$ \\
$\mathrm{No}$ & $\mathbf{n}(\%)$ \\
¿Considera que la automedicación es peligrosa? & $57(93)$ \\
$\mathrm{Si}$ & $4(7)$ \\
No & $\mathbf{n}(\%)$ \\
¿Cuenta con seguro médico? & $6(9,8)$ \\
$\mathrm{Si}$ & $55(90,2)$ \\
No & $\mathbf{n}(\%)$ \\
Consumo de medicamentos con bebidas alcohólicas & $57(93)$ \\
$\mathrm{Si}$ & $4(7)$ \\
No & \\
\hline
\end{tabular}

\section{DISCUSION}

La salud de una comunidad está relacionada, entre otros factores, con el consumo de medicamentos, lo que constituye una señal indirecta de la prevalencia de los problemas de salud. En nuestro estudio del total de participantes, el $97 \%$ de la población encuestada 
reportó consumir algún medicamento, de los cuales, el 53\% se automedicaba, esta cifra elevada es el resultado de la amplia diversidad de fármacos disponibles y lo extendido de su utilización. Su uso se ha generalizado, y son empleados para el tratamiento de enfermedades o para el alivio sintomático, e incluso, para la prevención de enfermedades o conservación de la salud.

Sin embargo, 53\% de automedicación es una cifra inferior al comparar con estudios realizados en diferentes países de Europa y América Latina. Así en un estudio llevado a cabo en estudiantes universitarios de Valencia - España la automedicación fue $91 \%{ }^{(3)}$. En estudiantes del Grado en Farmacia de la Universidad de Murcia - España fue $72,5 \%{ }^{(5)}$. Asimismo, en otro estudio en estudiantes de la Universidad de Egipto de la ciudad de Mansoura, fue $62,9 \%^{(10)}$.

Un estudio en inmigrantes latinoamericanos adultos de Sevilla- España ${ }^{(7)}$, reflejó un $77,4 \%$ de automedicación.

En Venezuela ${ }^{(8)}$ un $87 \%$ de estudiantes revelaron que se automedicaron tanto con medicamentos de venta libre como con los de venta con recetas. En Perú( ${ }^{(9)}$, en un estudio en personas adultas que acudieron a 22 farmacias, presentaron $88 \%$ de automedicación.

En un estudio transversal de base poblacional en 1820 adultos del Distrito Federal, Brasil $^{(11)}$, la prevalencia de automedicación fue de 14,9\%. Esta cifra muy diferente a las anteriores puede ser debido a la metodología.

A juzgar por los altos porcentajes de automedicación en la región, el presente estudio se encuentra con una prevalencia menor a la esperada, sin embargo, sigue siendo una cifra elevada teniendo en cuenta que el $88,5 \%$ de los funcionarios encuestados cuentan con seguro médico y que mismo en la empresa cuentan con un médico con el que pueden consultar dos veces por semana durante el horario laboral, esto puede ser atribuido a que muchas veces no consideran importante su dolencia y no es necesario consultar al médico o no quieren perder tiempo yendo a consultar.

En el estudio realizado se observa que las mujeres se automedicaron más (58\%) que los varones aunque la diferencia no es tan relevante. Este resultado coincide con estudios de otros países como Venezuela ${ }^{(8)}, \operatorname{Perú}^{(9)}, \operatorname{España}^{(5)}$ y Egipto ${ }^{(10)}$, donde la automedicación fue principalmente en mujeres.

La automedicación fue mayor entre los que poseen 20 a 30 años de edad y va disminuyendo en cuanto aumenta la edad de los entrevistados, lo que refleja, que los jóvenes son los que más utilizan medicamentos sin receta médica quizá porque en la era de la tecnología resulta más fácil acceder a internet para leer sobre los medicamentos que acudir al médico, o que con la edad uno se vaya haciendo más responsable en cuanto a su salud.

Los que trabajan en la empresa entre los 1 a 10 años se automedican más tal vez por que como los síntomas son repetitivos y ya anteriormente habían consultado con el médico deciden tomar el mismo medicamento pero ya sin receta médica.

Los síntomas más frecuentes que motivan a la automedicación son dolor de cabeza $(67,7 \%)$, seguido de dolor de garganta $(12,9 \%)$ y dolor de estómago $(9,7 \%)$, en coincidencia con estudio en Venezuela ${ }^{(8)}$ que refiere que los eventos adversos más frecuentes fueron los gástricos, vinculados con náuseas y dolores estomacales; seguidos por los eventos asociados con la presencia de somnolencia, con los dolores de cabeza. En un estudio en población adulta del distrito de Lima Metropolita- Perú(12), el motivo principal de la automedicación según síntomas fue síndrome doloroso. Asimismo, los síntomas por los cuales se automedicaron los estudiantes de la Universidad de Egipto de la ciudad de Mansoura ${ }^{(10)}$, fueron el resfriado $(70,1 \%)$, cefalea $(58,9 \%)$, dolor de garganta $(35,8 \%)$, cólico intestinal $(32,2 \%)$ y luego calambres $(31 \%)$. Son síntomas menores pero que realmente pueden ser causa de otras enfermedades más graves y que a la larga impidan reconocerlo y por lo mismo tratarlo adecuadamente.

Los medicamentos más consumidos por automedicación fueron los analgésicos/antiinflamatorios $(90,3 \%)$. Estos datos coinciden ampliamente con los comunicados en otros estudios $(5,8,9,11,13)$. Es conocida que la práctica generalizada de consumo de analgésicos y antiinflamatorios puede generar consecuencias para la salud debido a la presencia de reacciones adversas, tales como epigastralgia e hipertensión arterial $^{(12,14)}$.

Entre los motivos para llevar a cabo prácticas de automedicación la población estudiada menciona con mayor frecuencia que no consultan al médico por falta de tiempo $(58 \%)$ y 
otros no creen necesario consultar siempre al médico (42\%). Un estudio realizado en estudiantes de medicina de la ciudad de Medellín, el motivo principal para automedicarse y no consultar al médico fue por ahorrar tiempo que se gastan en los servicios de urgencias sin ser atendidos ${ }^{(15)}$. Otros estudios ${ }^{(5,10)}$ refieren que no hay necesidad de visitar al médico por una enfermedad menor.

La automedicación también parece ser una práctica frecuente en el entorno social de los funcionaros pues son los familiares y amigos quienes más influyen en la elección del medicamento con los que se realiza la automedicación. Un $42 \%$ de la población que participó del estudio afirma tener familiares y amigos que le sugirieron los medicamentos sin prescripción médica. Esta cifra es mayor que lo referido por Cecilia JM et $\mathrm{al}^{(5)}$, en un colectivo universitario, donde la mayoría $(58,33 \%)$ refirió automedicarse por conocimiento adquirido durante la carrera y solo un $28 \%$ por recomendación de un familiar o amigo. Cifras superiores al presente estudio, son referidos por Pillaca Medina ML et al ${ }^{(9)}$ y Helal RM et $\mathrm{al}^{(10)}(57,1 \%$ y $62,2 \%$ y, respectivamente). Estas diferencias podrían deberse a los diversos tipos de diseños.

La mayoría de los participantes de este estudio (74\%) que se automedicaban no consideraba importante la publicidad para escoger un medicamento. En un estudio en personas adultas del Distrito Jesús Nazareno, Perú( ${ }^{(9)}$ la publicidad en televisión fue el medio más influyente $(74,7 \%)$.

El $81 \%$ dice utilizar la información del prospecto. Cecilia $M J$ et al ${ }^{(5)}$ refiere que $40,1 \%$ utiliza el prospecto como fuente de obtención de información de medicamento. La diferencia puede ser debido a la forma de realizar las preguntas en los cuestionarios.

Aunque casi la totalidad de la población encuestada $(98 \%)$ considera valiosa la información que puede brindar el farmacéutico, sólo un 33\% declara recibir información del farmacéutico al adquirir un medicamento en la farmacia.

El $9,8 \%$ de los entrevistados admiten haber mezclado bebidas alcohólicas con medicamentos sin percibir el daño que podría causar esta mezcla a causa de las interacciones entre los mismos. La mayoria ( $89 \%$ ) tiene seguro médico y considera que la automedicación es peligrosa (93\%).

Investigaciones indican que pequeñas intervenciones educativas en poblaciones respecto a los riesgos de la automedicación generan cambios conductuales favorables incluso luego de transcurrido un año de la intervención ${ }^{(16,17)}$.

La automedicación es una práctica común en la población estudiada y en su entorno social inmediato. Otras conductas dan cuenta de que existe en la población abordada falta de información respecto a cómo deben utilizarse los medicamentos con seguridad y eficacia. La población estudiada se declara categóricamente receptiva al consejo del farmacéutico. Este escenario pareciera ideal para que el profesional farmacéutico, poniendo énfasis en la Atención Farmacéutica acentúe su rol principal de educador del paciente en todas las cuestiones que hacen al uso racional del medicamento, ocupando el lugar que le corresponde como miembro fundamental del equipo de salud, que es justamente el espacio que para él reclama la OMS. En ese sentido, es necesario que desde el órgano rector, se implementen estrategias educativas a nivel nacional con la colaboración de los farmacéuticos, con el fin de promover el uso responsable de medicamentos.

Agradecimientos: Agradecemos a las personas que participaron en la realización de las encuestas.

Financiación: Fondos propios del equipo de investigación.

Declaración: El presente trabajo, fue presentado en una jornada de jóvenes investigadores en modalidad de poster. Asimismo, declaramos la inexistencia, a nuestro juicio, de conflictos de intereses.

\section{REFERENCIAS BIBLIOGRÁFICAS}

1. Organización Mundial de la Salud. El papel del farmacéutico en el cuidado y la automedicación. Reporte de la 4o Reunión del Grupo Consultivo de la OMS sobre el papel del farmacéutico.1998. La Haya, Países Bajos. WHO/DAP/98.
2. Organización Mundial de la Salud. Nota descriptiva No338. Centro de prensa. Medicamentos: Uso racional de los medicamentos. 2010. (Internet). (Citado 20 mayo 2013). Disponible en: http://www.who.int/mediacentre/factsheets/fs 338/es/. 
3. Guillem Sáiz $P$, Francès Bozal F, Gimenez Fernandez F, Sáiz Sánchez C. Estudio sobre Automedicación en Población Universitaria Española. Rev Clin Med Fam. 2010; 3(2):99103.

4. García AJ, Alonso L, López P, Yera I, Ruiz A, Blanco N. Consumo de medicamentos referidos por la población adulta de Cuba. Rev Cuba Med Gen Integr. 2007;25(4):5-16.

5. Cecilia MJ, García-esta J, Atucha NM. La automedicación en estudiantes del Grado en Farmacia. Educ Med. 2018;19(5): 277-82.

6. Maidana GM, Lugo G, Vera $Z$, Pérez S, Mastroianni P. Evaluación de un programa de Atención Farmacéutica en pacientes con Diabetes Mellitus Tipo 2. Pharm Care España (Internet). 2016; 18(1): 3-15. Available from: http://www.pharmcareesp.com/index.php/Phar maCARE/article/view/259/235

7. González-López JR, Rodríguez-Gázquez MA L-CM. Automedicación en inmigrantes latinoamericanos adultos de Sevilla *. Acta Paul Enferm. 2012;25 (Número Especial 2):75-81.

8. Pablo MM De. La automedicación en la cultura universitaria Automedicación the culture in the university. Rev Invest (Guadalajara). 2011;73(35):219-39.

9. Pillaca-Medina ML, Carrión-Domínguez K. Automedicación en personas adultas que acuden a boticas del distrito Jesús Nazareno, Ayacucho 2015. An Fac med. 2016;77(4):38792.

10. Helal RM. Self-Medication in University Students from the City of Mansoura, Egypt. J Environ Public Health. 2017;1-7.
11. Domingues PHF, Galvao TF, Andrade KRC de, Araújo PC, Silva MT, Pereira MG. Prevalência e fatores associados à automedicação em adultos no Distrito Federal: estudo transversal de base populacional *. Epidemiol Serv Saude. 2017;26(2):319-30.

12. Rocío Hermosa-Moquillaza, César LozaMunarriz, Diana Rodriguez-Hurtado, César Arellano-Sacramento VH-M. Automedicación en un distrito de Lima. Rev Med Hered. 2016;27:15-21.

13. Machado-Alba JE, Echeverri-Cataño LF, Londoño-Builes MJ, Moreno-Gutiérrez PA, Ochoa-Orozco SA, Ruiz-Villa JO. Social, cultural and economic factors associated with selfmedication. Biomédica. 2014; 34: 580-8.

14. Ivet L, Ricardo S, Felipe F, Gárciga H, Farmacéuticas C, Policlínico I, et al. Reacciones adversas por antiinflamatorios no esteroideos Adverse reactions caused by non-steroidal antinflammatory drugs. Rev Cuba Farm. 2011; 45(1): 60-8.

15. Marie Clarie Berrouet Mejía MLR, Bernal DR. Automedicación de analgésicos y antibióticos en estudiantes de pregrado de medicina. Med UPB. 2017;36(2):115-23.

16. Maidana GM, Mastroianni PC, Vera $Z$, Samaniego L, Acosta $P$ LG. Impacto de la Atención Farmacéutica en la calidad de vida de pacientes con Diabetes Mellitus Tipo 2. Pharm Care España. 2016;18 (3):107-21.

17. Inmaculada Seva_Izquierdo MF-D, Martinez-Martinez F. Automedicación previa a la indicación farmacéutica en gripe o resfriado. VITAE. 2014; 21(1):30-7. 